\title{
Providing GFR Guarantees for TCP/IP Traffic over APON Access Systems
}

\author{
Sašo Stojanovski ${ }^{1}$, Maurice Gagnaire ${ }^{1}$, and Rudy Hoebeke ${ }^{2}$ \\ 1 E.N.S.T. InfRés, 46, rue Barrault, \\ 75634 Paris cedex 13, France \\ \{sassos, gagnaire\}@inf.enst.fr \\ 2 Alcatel Corporate Research Centre, \\ Francis Wellesplein 1, B-2018 Antwerpen, Belgium \\ rudy.hoebeke@alcatel. be
}

\begin{abstract}
The paper focuses on providing bandwidth guarantees for the Guaranteed Frame Rate (GFR) service category in the context of ATM-based Passive Optical Networks (APONs). Our work builds on the research performed on ATM multiplexers and extends it with the specifics of the APON access system. We study the performance of two known buffer management schemes (DFBA, WFBA), combined with either FIFO or per-VC queueing, when applied on virtual circuits carrying TCP/IP traffic. Our simulation results show that with fa irly small buffers at the Optical Network Units, the schemes based on per-VC queueing yield very good performance in large number of cases. The schemes based on FIFO queueing suffer from unfairness, although they do provide bandwidth guarantees in case the GFR bandwidth reservation is kept reasonably small. Tagging helps protecting TCP-like responsive traffic but does not prevent aggressive traffic from grabbing the nonreserved bandwidth. Tagging also increases unfairness.
\end{abstract}

\section{Introduction}

Guaranteed Frame Rate (GFR) is a new ATM service category intended to support non-real time applications that send data in the form of frames. Contrary to the Available Bit Rate (ABR) capability, the GFR service itself does not provide any explicit feedback to the traffic sources. Therefore, the traffic sources are supposed to be responsive to implicit congestion feedback information (dropped frames) and adapt their transmission rate. Since this is an IP-dominated world, the GFR service category will mostly be used for carrying TCP/IP traffic and the congestion control will consequently be performed via layer-4 mechanisms (TCP), rather than via layer-3 mechanisms (ABR).

The GFR service is defined in [1] with the following parameters: Peak Cell Rate (PCR) and its associated Cell Delay Variation (CDV) tolerance $\tau_{P C R}$, Minimum Cell Rate (MCR) and its associated CDV tolerance $\tau_{M C R}$, Maximum Burst Size (MBS) and Maximum Frame Size (MFS). The MCR parameter specifies the bandwidth guarantee that should be provided to the user under all 
network conditions. However, the user is allowed to send traffic well beyond this guarantee. In the latter case the netwo rk may perform some policing actions (tagging or discarding) on the submitted traffic. This is done on a frame-basis rather than cell-basis, which is the main originality of GFR with respect to the existing ATM service categories (notably the nrt-VBR with tagging).

Recently, there has been much interest in mechanisms for supporting the GFR service, notably: buffer management schemes and service disciplines. The former mechanism decides whether a frame is to be admitted into the buffer, whereas the latter determines the order in which the buffered cells will be transmitted.

It is well established (see 2]) that sophisticated per-VC service disciplines (e.g. Weighted Fair Queueing (WFQ) or Weighted Round Robin (WRR)) are sufficient for providing per-connection bandwidth guarantees in situations with infinite buffers, or in situations with traffic compliant to the Generic Cell Rate Algorithm (GCRA). However, if the sources are "elastic" in the sense that they can transmit at rates greater than those contracted (which is the case with GFR) and if the VCs carrying el astic traffic share the same buffer space (which is usually the case in all implementations), then the per-VC service disciplines must be complemented with active buffer management schemes in order to provide bandwidth guarantees. This argument is even stronger when the traffic carried along the ATM VCs is responsive to implicit feedback, as is the traffic generated by TCP sources.

Several GFR-enabling schemes have been investigated in the literature in the context of ATM multiplexers (see [3]), which are normally located at the output ports of ATM switches. Our research extends in the domain of APONs, taking into account their specifics.

An APON access system is shown in Figure 1 It is built on a passive optical tree, with a single Optical Line Termination (OLT) at the root and several Optical Network Units (ONU) at the leaves. It is destined for serving residential and small-business users. The number of ONUs connected to the optical tree is limited by the optical power budget, but the number of end-users may be much larger, since several end-users may be connected to a single ONU.

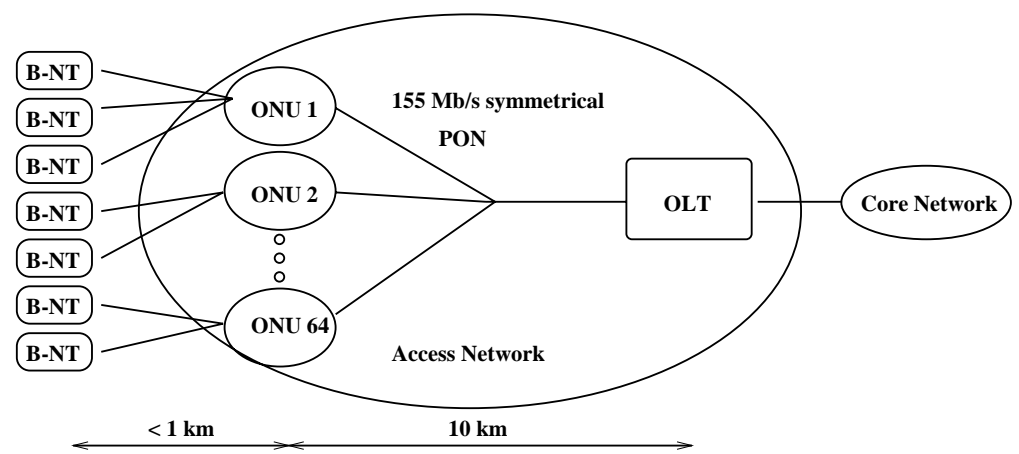

Fig. 1. The APON access system considered in this paper. 
Since the APON system is built on a shared medium, a MAC protocol is needed for controlling the access to the medium in the upstream direction. The ATM cells generated by the end-users are buffered at the ONUs. The latter are being periodically polled by the OLT for sending their bandwidth requests. The OLT then issues transmission permits and sends them in the downstream direction. Only upon a reception of a permit is the ONU allowed to transmit a cell. An ideal MAC protocol should be completely transparen t to the ATM layer and the whole APON system should appear as an ATM multiplexer. However, this is only partially true for the following reasons: first, the request/permit mechanism introduces an inherent delay (round-trip time of $0.1 \mathrm{~ms}$ in our case); second, the system is hierarchical with aggregation occurring at the upper stage, and third, the buffer management schemes are performed at the input (the ONUs) rather than the output of the multiplexer.

To our knowledge this is the first study that investigates the performance of TCP/IP traffic carried accross APONs. And although the research presented here focuses on a specific kind of ATM access networks, we believe that some of the conclusions hold in general, especially those concerning the use of tagging for protection of TCP-like responsive flows.

In Section 2 we define the buffer management and queueing schemes which we consider for use at the ONU. Then, in Section 3 we propose two scenarios which are investigated in Section 4 by means of simulations. Section 5 summarises our findings.

\section{Buffer Management and Queueing Schemes}

In our study we consider a symmetrical $155.52 \mathrm{Mbit} / \mathrm{s}$ APON with 64 ONUs and frame structure as defined in 4]. Although the APON is an example of hierarchical multiplexer, sophisticated hierarchical queueing schemes, such as the one described in [5], cannot be used due to the geographical separation between the two stages. Therefore, two different and independent queueing schemes are performed at each stage. The OLT schedules the bandwidth requests sent by the ONUs using the Weighted Round-Rob in (WRR) discipline, defined in [6]. The service is performed on per-ONU rather than per-VC basis, since the OLT has no per-VC visibility. The weights allocated to each ONU are proportional to the aggregated MCR sum of all VCs stemming from the same ONU. On the other side, the ONU has the choice of using either the same WRR discipline, but this time on a per-VC basis, or do FIFO queueing.

We consider two known buffer management schemes at the ONU: Weighted Fair Buffer Allocation (WFBA) and Differential Fair Buffer Allocation (DFBA), defined in 7] and [8], respectively. Both of them make use of two global thresholds: $L B O$ and $H B O$, standing for Low and High Buffer Occupancy. A buffer allocation weight is associated to each $\mathrm{VC}$, and typically this weight is proportional to the connection's MCR. Untagged frames $(\mathrm{CLP}=0)$ are always accepted if the global buffer occupancy $X$ is lower than $L B O$. The differences between the two algorithms appear when the global buffer occupancy is between the two 
thresholds $(L B O<X<H B O)$. Both schemes use an admission criterion which is a function of the global and individual buffer occupancies, as well as the allocated weights. The frames which do not pass the admission criterion in WFBA are dropped in a deterministic manner, whereas in DFBA they are dropped with a probability which is also a function of the above-mentioned criteria. Note that probabilistic dropping is also used in Random Early Detection (RED), the most widely deployed buffer management scheme in today's Internet (see [9]).

In its original version WFBA does not distinguish between tagged and untagged frames. However, this extension is straightforward: tagged frames in both schemes are admitted into the buffer only if the global buffer occupancy $X$ is lower than $L B O$.

Two conformance definitions have been defined for the GFR service: GFR.1 and GFR.2. The difference between the two is that GFR.2 allows the network to tag the excess traffic using the $F-G C R A(T, f)$ algorithm, where $T=\frac{1}{M C R}$ and $f \geq(M B S-1) \cdot\left(\frac{1}{M C R}-\frac{1}{P C R}\right)$. Note, however, that tagging can also be virtual i.e. frames can be tagged internally at the ONU, without actually modifying the CLP bit (see 10]). The virtual tagging allows us to take a unified a pproach for both GFR.1 and GFR.2 conformance definition.

By combining the two buffer management schemes with FIFO or per-VC queueing, we obtain the following four schemes: WFBA+FIFO, DFBA+FIFO, $\mathrm{WFBA}+\mathrm{WRR}$ and DFBA+WRR. We investigate their performances by means of simulations in the following section.

\section{Simulation Scenarios}

We consider two heterogeneous scenarios referred to as: RESP1 and RESP2. In both scenarios we consider 32 GFR VCs carrying 10 TCP-NewReno connections each. The total number of TCP connections equals 320. Only 8 ONUs (out of 64) are active and each one is traversed by four GFR VCs. The VCs do not have equal MCR reservations, neither RoundTrip Times (RTT). The two scenarios differ in the way the MCRs and RTTs are distributed accross the ONUs. We tried to cover as many cases as possible. The parameters which a re common and specific to the two scenarios are given in Table 1 and Table 2, respectively. Table 1 contains the TCP-specific parameters, such as: version, Maximum Segment Size or timer granularity. It also shows the ONU buffer threshold settings ( $L B O$ and $H B O$ ) which are used for buffer management. Table 2 explains the MCR and RTT distribution accross the $32 \mathrm{VCs}$ and eight active ONUs.

The scenario-specific parameters (i.e. MCR reservations and RTT) for scenarios RESP1 and RESP 2 are illustrated in Figure 2 and Figure 3, respectively. Figures 4(a) and 4(b) illustrate the bandwidth-delay product for scenario RESP1 and RESP2, respectively, expressed in cells. In these, as well as in all subsequent figures, the abscissa values identify the active ONUs.

We use the NewReno version of TCP which is described in [11]. This is a bug-fixed version of TCP Reno which improves the performance of the latter in 


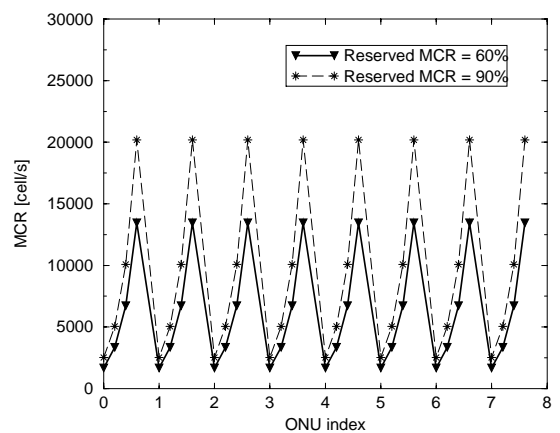

(a) GFR reservation $\left(M C R_{i}\right)$

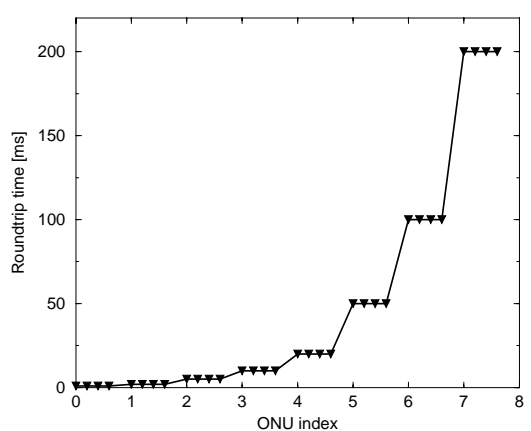

(b) Roundtrip time

Fig. 2. Scenario RESP1.

case of multiple segments dropped from a window of data. All 320 TCP sources are persistent i.e. they have always data for transmission.

\section{Simulation Results}

The simulations reported in this paper correspond to 30 seconds of simulated time. The results are expressed via the normalised goodput received by each VC, defined as: $R=\frac{\text { Goodput }_{i}}{M C R_{i}} \cdot R=1.0$ means that $V C_{i}$ has realised goodput which is exactly equal to its reservation $M C R_{i}$, without receiving any excess bandwidth. Similarly, $R=2.0$ means that $V C_{i}$ has realised goodput which is equal to twice its reservation and $R=0$ means that $V C_{i}$ has not realised any goodput a t all.

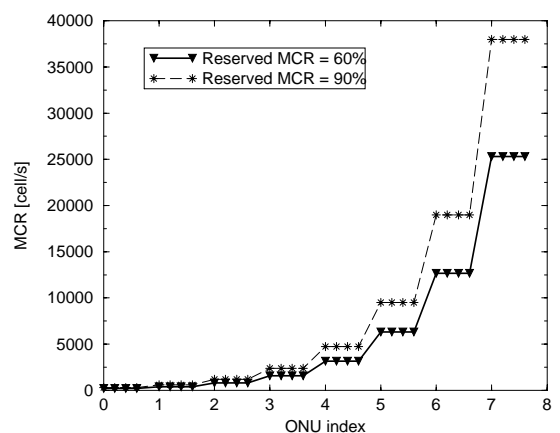

(a) GFR reservation $\left(M C R_{i}\right)$

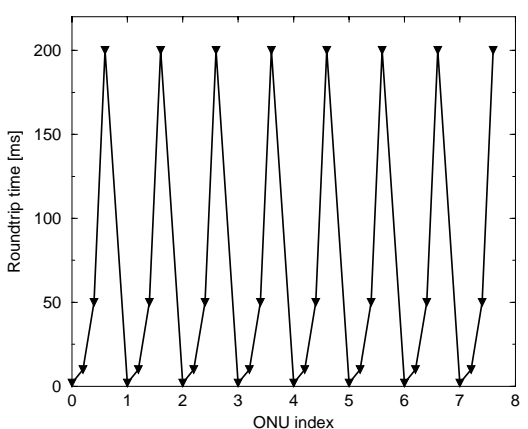

(b) Roundtrip time

Fig. 3. Scenario RESP2. 


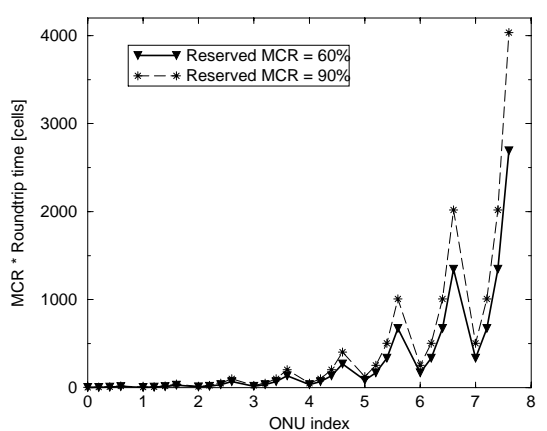

(a) Scenario RESP1

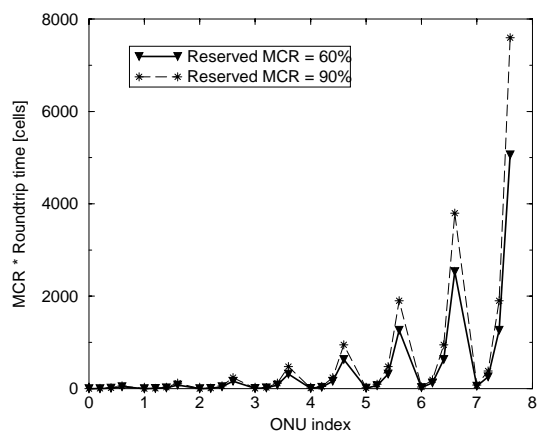

(b) Scenario RESP2

Fig. 4. Bandwidth-delay product.

Table 1. Common parameters for scenarios RESP1 and RESP2

\begin{tabular}{ll}
\hline Active ONUs & 8 (out of 64$)$ \\
GFR connections per ONU & 4 \\
TCP connections per VC & 10 \\
Total MCR reservation & either $60 \%$ or $90 \%$ of the system's capacity \\
TCP Max Segment Size (MSS) & 1460 octets \\
Maximum Frame Size (MFS) & 32 cells (This corresponds to the chosen MSS \\
& with all the overhead: 20 octets (TCP) + 20 \\
& (IP) +8 (LLC/SNAP) +8 (CPCS-AAL5) \\
& + padding.) \\
ONU buffer size & 2000 cells \\
LBO (HBO) threshold & $900(1800)$ cells \\
TCP version & NewReno \\
TCP timer granularity & $10 \mathrm{~ms}$ \\
\hline
\end{tabular}

In subsection 4.1 we consider scenarios with responsive traffic only. No tagging is used. Then, in subsection 4.2 we evaluate the TCP performance in presence of non-responsive and very aggressive traffic. The conclusion of 4.2 is that tagging has to be used. Therefore, in subsection 4.3 we revisit the responsive traffic scenario from 4.1, but this time with tagging being applied.

\subsection{Responsive Traffic Only and No Tagging}

Figure 5 illustrates the normalised goodput for schemes relying on either FIFO or WRR queueing, for scenario RESP1. The global GFR reservation (i.e. the sum of per-connection $M C R_{i}$ ) equals either $60 \%$ or $90 \%$ of the system capacity. Also shown in the figures are three horizontal lines. The first one, $y=1.0$, represents the lowest value for the normalised goodput at which the bandwidth guarantee 
Table 2. Specific parameters for scenarios RESP1 and RESP2

\begin{tabular}{|c|c|c|}
\hline & $R E S P 1$ & $R E S P \mathscr{2}$ \\
\hline $\begin{array}{l}M C R \text { for VCs at } \\
\text { the same } O N U\end{array}$ & $\begin{array}{l}\text { relate to each other as } 1: 2: 4 \\
: 8\end{array}$ & $\begin{array}{l}\text { relate to each other as } 1: 1: 1 \\
: 1\end{array}$ \\
\hline $\begin{array}{l}\text { Aggregated } M C R \\
\text { of the eight ac- } \\
\text { tive ONUs }\end{array}$ & $\begin{array}{l}\text { relate to each other as } 1: 1: 1 \\
: 1: 1: 1: 1: 1\end{array}$ & $\begin{array}{l}\text { relate to each other as } 1: 2: 4 \\
: 8: 16: 32: 64: 128\end{array}$ \\
\hline Roundtrip time & $\begin{array}{l}\text { same for VCs at the same } \\
\text { ONU; changes from one ONU } \\
\text { to another as: } 1: 2: 5: 10: 20 \\
: 50: 100: 200 \mathrm{~ms}\end{array}$ & $\begin{array}{l}2: 10: 50: 200 \mathrm{~ms} \text { for ev- } \\
\text { ery four consecutive VCs; the } \\
\text { same pattern is repeated at } \\
\text { each ONU }\end{array}$ \\
\hline
\end{tabular}

is still met. The other two lines $(\mathrm{y}=1.66$ and $\mathrm{y}=1.11)$ correspond to the ideal value for $\mathrm{t}$ he normalised goodput, for which every $\mathrm{VC}$ gets a share of the available (non-reserved) bandwidth in proportion to its MCR. (Note: $1.66=\frac{100}{60}$ and $1.11=\frac{100}{90}$.)

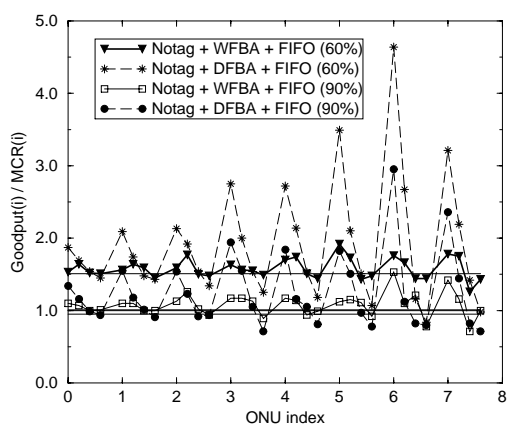

(a) FIFO

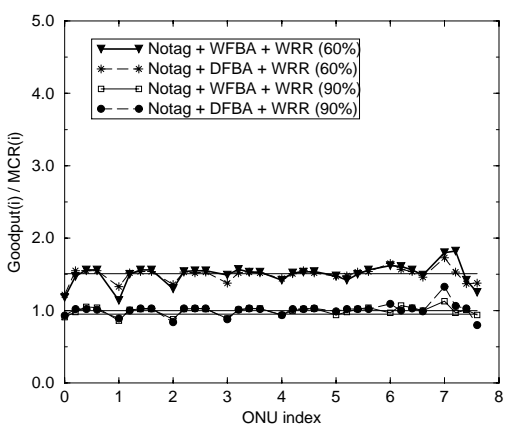

(b) WRR

Fig. 5. RESP1: normalised TCP goodput.

As seen from Figure 5, when the global GFR reservation equals 60\%, all VCs attain their guarantees $(R>1.0)$. When FIFO queueing is used, the available bandwidth is not fairly distributed since low-rate VCs at each ONU get a higher proportion of available bandwidth (the curves contain ripples). This is especially striking with the DFBA scheme. When the global reservation equals $90 \%$, several VCs fail to achieve their guarantee, especially with the DFBA scheme. On the other hand, both buff er management schemes perform equally well when WRR queueing is used. Almost all VCs attain their guarantee and free bandwidth is distributed ideally (almost flat curves). 


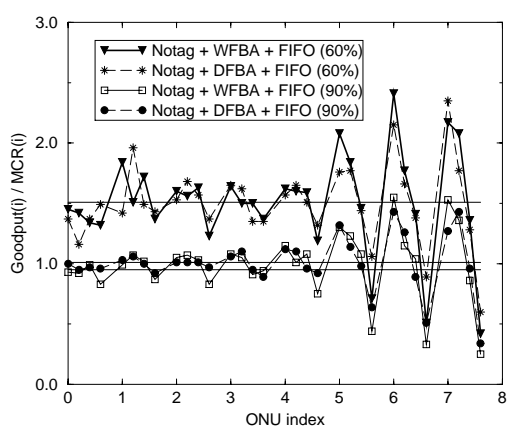

(a) FIFO

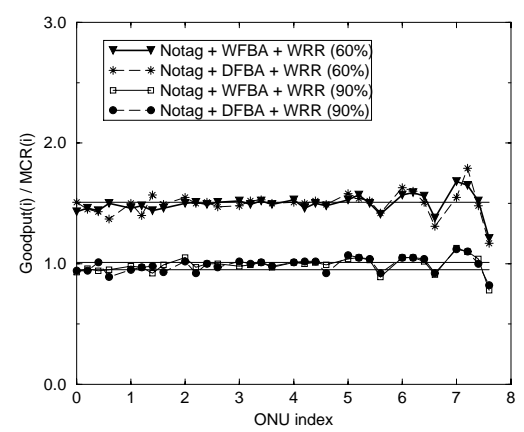

(b) WRR

Fig. 6. RESP2: normalised TCP goodput.

Figure 6illustrates the performance of FIFO and WRR based schemes in scenario RESP2. Again one sees that WRR schemes are fairer than FIFO schemes in distribution of free bandwidth. What is new is that there is no striking difference between WFBA and DFBA when FIFO queueing is used. This is probably due to the fact that VCs belonging to the same ONU in scenario RESP2 have equal MCR reservations.

It can further be noted from Figure 6 that even at $60 \%$ reservation there are several connections that do not meet the guarantee when FIFO queueing is used. This is explained by the fact that the last two ONUs contain VCs whose bandwidth-delay product is greater than the ONU buffer size (much greater than in scenario RESP1). On the other hand, the WRR schemes (see again Figure 6) have no problems in meeting the guarantee at $60 \%$ reservation. Furthermore, they succeed to make it within $15 \%$ of the MCR guarantee at $90 \%$ reservation, even for the most extreme case (the fourth $\mathrm{VC}$ from $\mathrm{ONU}_{7}$ ).

Curiously enough, the probabilistic dropping in DFBA does not yield better performance than the deterministic dropping of WFBA. This may be counterintuitive, since the field trials with RED have shown the benefits of probabilistic dropping when carrying TCP traffic in today's Internet. We see two possible explanations for this observation. First, the ATM VCs in our scenarios carry several TCP connections, so even if frames in WFBA are dropped deterministically, they do not necessarily belong to the same T CP connection. Second, and more importantly, the RED scheme reacts on the average rather than instantaneous queue size as in DFBA. Hence, even if DFBA drops frames probabilistically, there is still a non-negligeable probability of "hitting" the same TCP connection when the global occupancy temporarily persists at higher values. Given this remark, we shall consider only the WFBA scheme in the remainder of this paper. 


\subsection{TCP Performance in Presence of Non-Responsive Traffic}

It is easy to show that without tagging, the buffer management schemes themselves are not sufficient for protecting the responsive traffic from the aggressive one. Tagging is an effective way for preventing the aggressive sources from occupying large portion of the shared buffer space. The network elements operating at the ATM layer (e.g. ONUs) have no higher layer visibility and, hence, do not discriminate against responsive and non-responsive traffic (e.g. TCP from UDP). Even if that were the case, the ON U must still not rely on the higher-layer information, since there may be faulty or deliberately aggressive TCP implementations out there. Therefore, tagging should systematically be applied on all traffic.

In this subsection we consider a scenario with non-responsive greedy sources and we refer to it as NONRESP2. Scenario NONRESP2 is obtained from RESP2 by substituting the first $\mathrm{VC}$ at every active $\mathrm{ONU}$ with a VC carrying nonresponsive traffic. The latter is modelled as a constant bitrate flow carrying 32cell frames at $155.52 \mathrm{Mbit} / \mathrm{s}$ (measured at the physical layer). Thus the entire system is at extreme overload.

When tagging is applied, there is a choice to be made for the MBS parameter in the F-GCRA function. Several approaches for the MBS exist in the literature. For instance, 12] proposed a default value of 192 cells, whereas [10] proposed to compute it as a function of the MCR and the number of TCP connections in a VC. We propose to use a fixed and rather large value of: $M B S=200 \cdot M F S$, regardless of the connection's MCR.

Figure 7 shows the TCP throughput of the responsive connections in case WFBA+FIFO (or WFBA+WRR) with tagging is used, at both $60 \%$ and $90 \%$ reservation. The figure shows that with the use of tagging the responsive sources can be protected from the non-responsive ones as far as the MCR guarantee is concerned. The only conections that fail to meet their guarantee are those with extremely large bandwidth-delay product, and this should not be attributed to the presence of aggressive traffic.

However, the Figure 7 also shows that the normalised goodput curves are collapsed around $\mathrm{y}=1.0$. This means that, while the bandwidth guarantee is met in the majority of cases, the free bandwidth has entirely been grabbed by the aggressive connections. This is clearly visible in Figure 8 which shows the MCR and the realised throughput for each VC (including the aggressive ones). One sees that the greedy connections have realised much better throughput than the responsive o nes.

\subsection{Responsive Traffic with Tagging}

Subsection 4.2 highlighted the importance of tagging. Since the ONUs have no higher layer visibility, they should perform tagging on all traffic. Next we revisit the case with TCP traffic only, which was considered in subsection 4.1, but this time we investigate the impact of tagging, even in absence of non-responsive traffic. Because of lack of space, only RESP2 scenario and WFBA scheme are considered. 


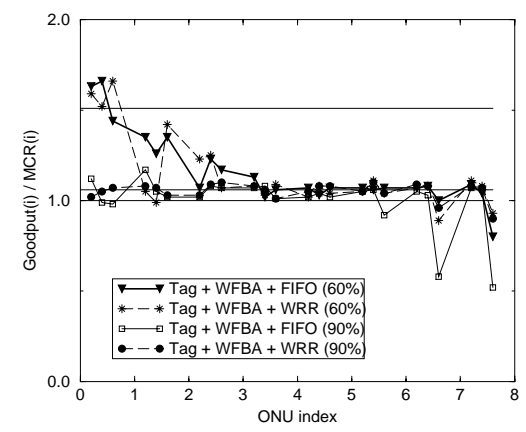

Fig. 7. NONRESP2: normalised TCP goodput of responsive VCs under the WFBA+FIFO and WFBA+WRR schemes.

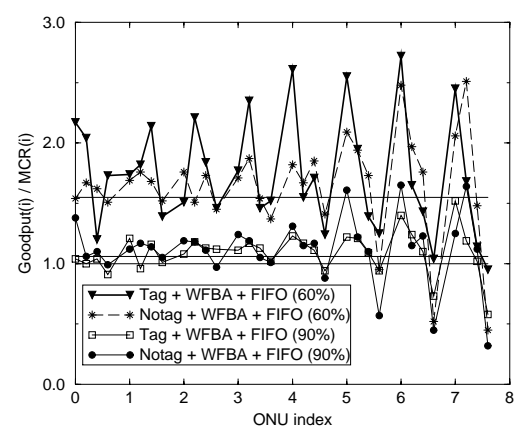

(a) Tagging + FIFO

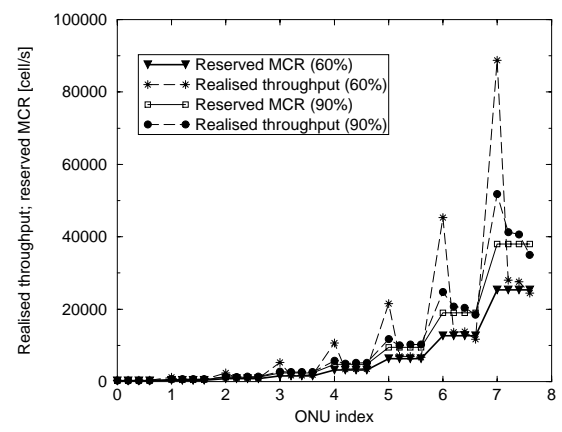

Fig. 8. NONRESP2: realised goodput of both responsive and aggressive VCs under the WFBA+WRR scheme.

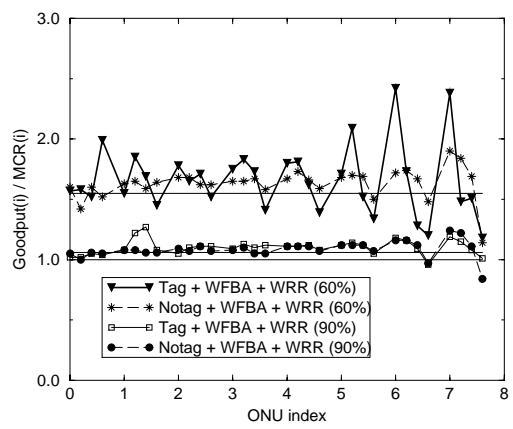

(b) Tagging + WRR

Fig. 9. RESP2: normalised TCP goodput with tagging.

Figure 9 shows the VC goodput when tagging is used in addition to either WFBA+FIFO or WFBA+WRR. The curves corresponding to the non-tagged case (Figure 6) are also reproduced for comparison. The figures show that at high GFR reservation there is no significant difference whether tagging is used or not. However, at $60 \%$ reservation one sees that the distribution of free bandwidth with tagging becomes less fair (indicated by greater ripples in the normalised throughput curves). T his is especially apparent when WRR queueing is used.

\section{Conclusions}

In this paper we have considered two buffer management schemes (WFBA and DFBA) combined with two queueing schemes (FIFO and WRR) in order to 
provide GFR guarantees to TCP/IP traffic over APON access networks. We find out that:

- at reasonably small reservations (60\% of the system capacity), all schemes allow the VCs to attain their guarantees, with the exception of some VCs with extremely high bandwidth-delay product in the FIFO schemes;

- at very high GFR reservation (90\% of the system capacity), the high-rate VCs at several ONUs fail to meet their guarantee in the FIFO schemes, whereas in the WRR schemes this happens only for VCs with extremely high bandwidth-delay product;

- the FIFO-based schemes are intrinsically unfair in free bandwidth distribution, since low-rate VCs get larger proportion of excess bandwidth;

- the schemes with per-VC queueing distribute the free bandwidth in a very fair manner, except for VCs with extremely high bandwidth-delay product;

- the probabilistic dropping of DFBA does not bring any performance gain compared to the less complicated WFBA scheme;

- tagging is necessary in order to isolate responsive traffic from aggressive one;

- tagging increases unfairness in absence of non-responsive traffic, and

- tagging does not prevent aggressive traffic from grabbing all the free bandwidth.

Referring to the last conclusion, it would be interesting to investigate whether the three-colour tagging, which is currently considered in the diffserv community (see [13]) would help. Although the ATM cell may have only two colours $(\mathrm{CLP}=0$ or $\mathrm{CLP}=1)$, three colours could still be implemented via virtual tagging.

\section{Acknowledgements}

This work has been sponsored by Alcatel CRC in Antwerp-Belgium within a research contract with ENST.

\section{References}

1. ATM Forum's Traffic Management Specification 4.1, ATM Forum af-tm-0121.000 (1999)

2. B.Suter, T.V.Lakshman, D.Stiliadis, A.Choudhury, "Efficient Active Queue Management for Internet Routers", Networld+Interop98 Engineers Conference Notes, Las Vegas (1998)

3. Olivier Bonaventure, "Providing bandwidth guarantees to internetwork traffic in ATM networks", Proceedings on ATM '98 Workshop, Fairfax (1998)

4. ITU-T Recommendation G.983, "High Speed Optical Access Systems Based on Passive Optical Network (PON) Techniques" (1998)

5. J.C.R. Bennet and H.Zhang, "Hierarchical Packet Fair Queueing Algorithms", IEEE/ACM Transactions on Networking, vol.5, no.5 (1998)

6. Manolis Katevenis, Stefanos Sidiropoulos and Costas Courcoubetis, "Weighted Round Robin Cell Multiplexing", IEEE JSAC, vol.9, no.8 (1991) 
7. Juha Heinanen and Kalevi Kilkki, "A Fair Buffer Allocation Scheme", Computer Communications, vol.21, no.3 (1998)

8. Rohit Goyal, "Buffer Management for the GFR Service", ATM Forum contribution af98-0405 (1998)

9. Braden, Clark et al., "Recommendation on Queue Management and Congestion Avoidance in the Internet", IETF RFC 2309 (1998)

10. Byoung-Joon Lee, "GFR Dimensioning for TCP Traffic", ATM Forum contribution af98-0271 (1998)

11. Tom Henderson and Sally Floyd, "The NewReno Modification to TCP's Fast Recovery Algorithm", Internet Draft draft-ietf-tcpimpl-newreno (1998)

12. Juha Heinanen, "GFR Signalling", ATM Forum contribution af98-0024 (1998)

13. Juha Heinanen, Roch Guerin, "A Three-Colour Marker", Internet Draft draftheinanen-diffserv-tcm-01.txt (1999) 